\title{
Augmentation Pressure Point P1
}

National Cancer Institute

\section{Source}

National Cancer Institute. Augmentation Pressure Point P1. NCI Thesaurus. Code C122085.

The first aortic systolic pressure peak, also referred to as a forward wave, used to calculate the aortic augmentation index. 